\title{
Health Risk Assessment of Banned Veterinary Drugs and Quinolone Residues in Shrimp through Liquid Chromatography-Tandem Mass Spectrometry
}

\author{
Ming-Yang Tsai ${ }^{1,2, \dagger}$, Chuen-Fu Lin ${ }^{3, \dagger}$, Wei-Cheng Yang ${ }^{4}$, Chien-Teng Lin ${ }^{3}$, Kuo-Hsiang Hung ${ }^{2}$ \\ and Geng-Ruei Chang ${ }^{3, *}$ \\ 1 Animal Industry Division, Livestock Research Institute, Council of Agriculture, Executive Yuan, \\ Tainan 71246, Taiwan; mytsai@mail.tlri.gov.tw \\ 2 Graduate Institute of Bioresources, National Pingtung University of Science and Technology, \\ Pingtung 91201, Taiwan; khhung424@mail.npust.edu.tw \\ 3 Department of Veterinary Medicine, National Chiayi University, Chiayi 60054, Taiwan; \\ cflin@mail.ncyu.edu.tw (C.-F.L.); vet540423@gmail.com (C.-T.L.) \\ 4 Department of Veterinary Medicine, School of Veterinary Medicine, National Taiwan University, \\ Taipei 10617, Taiwan; yangweicheng@ntu.edu.tw \\ * Correspondence: grchang@mail.ncyu.edu.tw; Tel.: +886-5-2732959; Fax: +886-5-2732917 \\ + These authors contributed equally to this work.
}

Received: 24 May 2019; Accepted: 13 June 2019; Published: 17 June 2019

check for updates

\begin{abstract}
The presence of antibiotic residues in seafood and their effect on public health constitute a matter of concern for consumers worldwide. Antibiotic residues can have adverse effects on both humans and animals, especially residues of banned veterinary drugs. In this study, we applied a validated method to analyze veterinary drug residues in shrimp, including the levels of banned chloramphenicol, malachite green, leucomalachite green, and four nitrofuran metabolites as well as thiamphenicol, florfenicol, and five quinolones, which have no recommended maximum residual levels in shrimp tissues in Taiwan. We collected 53 samples of whiteleg, grass, or giant river shrimp from Taiwanese aquafarms and production areas from July 2016 to December 2017 . We found $0.31 \mathrm{ng} / \mathrm{g}$ of a chloramphenicol in one grass shrimp, $5.62 \mathrm{ng} / \mathrm{g}$ of enrofloxacin in one whiteleg shrimp, $1.52 \mathrm{ng} / \mathrm{g}$ of flumequine in one whiteleg shrimp, and $1.01 \mathrm{ng} / \mathrm{g}$ of flumequine in one giant river shrimp, indicating that $7.55 \%$ of the samples contained veterinary drug residues. We evaluated the health risk by deriving the estimated daily intake (EDI). The quinolone residue EDI was below $1.0 \%$ of the acceptable daily intake recommended by the United Nations Food and Agriculture Organization and World Health Organization. The risk was thus discovered to be negligible, indicating no immediate health risk associated with shrimp consumption. The present findings can serve as a reference regarding food safety and in monitoring of the veterinary drug residues present in aquatic organisms. Continual monitoring of residues in shrimp is critical for further assessment of possible effects on human health.
\end{abstract}

Keywords: veterinary drug; residue; shrimp; mass spectrometry; risk assessment

\section{Introduction}

Taiwan has a geographic location and environment conducive to aquaculture development. Aquaculture in Taiwan has a long history of more than three centuries, and it has rapidly expanded, diversified, intensified, and technologically advanced from 1960 to the 1990s [1]. Despite Taiwan's land and water resource limitations, it is one of the major aquaculture producers in the world; therefore, it was once called the "kingdom of aquaculture" [2]. Until now, over 35 major and candidate species have been cultured for commercial purposes [3]. The average revenue of Taiwan from 
aquaculture reached US $\$ 11$ billion between 2010 and 2015 [3]. Moreover, the annual aquaculture production was approximately $300,000 \mathrm{t}$ during the 2010s. Specifically, shrimp culture production has been notable because Taiwan's government has strongly supported aquaculture since the 1980s [4], in particular, aquaculture of shrimp-including whiteleg shrimp (Litopenaeus vannamei), grass shrimp (Penaeus monodon), giant river shrimp (Macrobrachium rosenbergii), sand shrimp (Metapenaeus ensis), and kuruma shrimp (Penaeus japonicus). Moreover, the average revenue from the shrimp aquaculture industry reached US $\$ 3000$ million between 2010 and 2016 .

Intensive and large-scale breeding is preferred by aquaculture farmers in Taiwan because the farmers are limited to land use [5]. Moreover, their farms are generally situated near residential and agricultural areas, which makes biological control difficult [6]. The cultured species became more susceptible to bacterial, viral, parasitical, and fungal infections, necessitating the use of various veterinary drugs for the prevention and treatment of these infections. However, when such drugs are heavily employed in aquaculture, aquaculture products may contain drug residues, potentially exposing the consumers of the products to these residues. This has an inadvertent ecological impact and raises health concerns such as increased risk of allergies, carcinogen exposure, and the development of bacteria resistant to antibiotics [5]. Because of their benthic feeding behavior, shrimp can be used to indicate the levels of chemicals in aquatic environments [7]. Analyzing the amounts of pollutants in shrimp indicates the environmental levels of veterinary drugs and the extent to which drugs are transferred through the trophic chain.

In Taiwan, shrimp are the crustaceans cultured most extensively in land-based ponds [3], and in the inner regions of the island, shrimp are generally bred in a mix with other aquatic products. Typically, veterinary drugs are used to prevent or treat diseases in nonshrimp targets, which pollutes water and soil environments while contaminating shrimp. In this regard, shrimp products have played a crucial role in seafood safety. Residues of veterinary drugs in shrimp are a crucial concern regarding public health, especially when the residues are of banned chemicals that have been employed illegally. Therefore, in this study, we detected in shrimp samples the levels of the following banned veterinary compounds in Taiwan: leucomalachite green (LMG), malachite green (MG), nitrofuran metabolites, and chloramphenicol. These compounds' maximum residue limits (MRLs) in shrimp have not been established by the Taiwan Food and Drug Administration (TFDA). In addition, we detected florfenicol, thiamphenicol, and quinolone residues, including danofloxacin, difloxacin, enrofloxacin, flumequine, and sarafloxacin, in shrimp. The TFDA defined the MRL levels of these quinolones in livestock and chicken in 2018; however, the use of these compounds in the cultivation of shrimp is banned. The present study's detection of the residues of these compounds in shrimp thus indicates the degree of legal compliance regarding the use of these products. In addition, the seafood-consumption-based estimated daily intake (EDI) of these contaminants was determined to identify the effects of exposure to these veterinary drugs on the health of the Taiwanese public. Results were applied for assessing the risk of exposure to veterinary drugs among consumers in Taiwan. The findings of this study are useful when conducting evaluations of seafood safety and may be used as a reference among health authorities for establishing regulations.

\section{Materials and Methods}

\subsection{Samples}

Shrimp samples were obtained between July 2016 and December 2017 from aquafarms in the principal production areas (Yunlin, Chiayi, Tainan, Kaohsiung, and Pingtung). In total, 53 samples (23 whiteleg, 16 grass, and 14 giant river shrimps) were collected. These shrimps are bred on a large scale in Taiwan [3]. We removed, homogenized, and stored the soft tissues of all shrimp samples at $-20^{\circ} \mathrm{C}$ until they were analyzed. 


\subsection{Chemicals and Reagents}

Analytical compounds included veterinary drugs, namely chloramphenicol $(98.6 \%)$, thiamphenicol (98.5\%), florfenicol (98\%), MG (98.0\%), and LMG (99.0\%), purchased from Dr. Ehrenstorfer GmbH (Ausburg, Germany). Nitrofuran metabolites, namely 5-methylmorpholino-3-amino-2-oxazolidinone (AMOZ, 99.9\%), 3-amino-2-oxazolidinone (AOZ, 99.7\%), and 1-aminohydantoin hydrochloride (99.9\%), were obtained from Sigma-Aldrich (St. Louis, MO, USA). Semicarbazide hydrochloride (99.5\%) was obtained from Chem Service Inc. (West Chester, PA, USA). Danofloxacin $(98.0 \%)$, difloxacin $(98.0 \%)$, enrofloxacin (98.0\%), flumequine (98.0\%), and sarafloxacin (95.0\%) were obtained from Sigma-Aldrich. In addition, stable isotopically labeled internal standards, AMOZ-D5 (99.0\%) and AOZ-D4 (99.0\%), were purchased from Dr. Ehrenstorfer GmbH. Other internal standards, namely SC-13C15N2 (99\%), MG-D5 picrate (99.9\%), and LMG-D5 (99.8\%), were purchased from Sigma-Aldrich.

Chromatography-grade acetonitrile ( $\mathrm{ACN}$ ), acetone, ammonium acetate, dipotassium phosphate, ethyl acetate (EtOAc), formic acid (FA), hydrochloric acid $(\mathrm{HCl})$, methanol $(\mathrm{MeOH})$, n-hexane, and sodium hydroxide $(\mathrm{NaOH})$ were purchased from Merck (Darmstadt, Germany). Reagent-grade 2-nitrobenzaldehyde (2-NBA), sodium chloride ( $\mathrm{NaCl}$ ), and $N, N, N^{\prime}, N^{\prime}$-tetramethyl-1,4-phenylenediamine dihydrochloride (TMPD) were purchased from Sigma-Aldrich.

\subsection{Instruments and Apparatus}

A vortex mixer (type 37600 mixer, Barnstead/Thermolyne, Dubuque, IA, USA), a centrifuge (Allegra X-22R, Beckman Coulter, Fullerton, CA, USA), a nitrogen evaporator (N-Evap-111, Organomation Associates Inc., Berlin, Germany), and a nitrogen generator (Model 05B, System Instruments Co., Tokyo, Japan) were used to prepare samples. The liquid chromatography-tandem mass spectrometry (LC/MS-MS) apparatus comprised an LC system (Agilent Technologies 1200, Agilent Technologies, Palo Alto, CA, USA) and a mass spectrometer (ABI 4000 QTRAP, Applied Biosystems, Foster City, CA, USA). To determine the levels of residues of chloramphenicol classes, nitrofuran metabolites, and quinolone classes in samples, chromatographic separation was performed in an analytical column (Chromolith Performance RP-18e, $100 \mathrm{~mm} \times 3 \mathrm{~mm}$, Merck, Darmstadt, Germany) and a guard column (Chromolith Guard Column RP-18e, $5 \mathrm{~mm} \times 4.6 \mathrm{~mm}$, Merck). In addition, MG and LMG were separated using a Purospher STAR RP-18 endcapped analytical column $(100 \mathrm{~mm} \times 2.1 \mathrm{~mm}$ $\times 2 \mu \mathrm{m}$, Merck) and Purospher Star RP-18 endcapped guard column (4 mm $\times 4 \mathrm{~mm} \times 5 \mu \mathrm{m}$, Merck).

\subsection{Analysis of LC/MS-MS Conditions}

An injection volume of $10 \mu \mathrm{L}$ was used for determining the levels of chloramphenicol classes, MG, LMG, and quinolone classes and $20 \mu \mathrm{L}$ for nitrofuran metabolites. Chloramphenicol, thiamphenicol, and florfenicol levels were analyzed through gradient elution by using the A1 eluent $(0.1 \% \mathrm{MeOH})$ and $\mathrm{B} 1$ eluent $(100 \% \mathrm{MeOH})$. A mobile phase gradient was started at $40 \% \mathrm{~B} 1$ for $1 \mathrm{~min}$ at a flow rate of $0.5 \mathrm{~mL} / \mathrm{min}$, linearly increased to $90 \% \mathrm{~B} 1$ at $4 \mathrm{~min}$, and subsequently maintained constant until $6 \mathrm{~min}$. Thereafter, it was changed to $40 \%$ B1 after $6.1 \mathrm{~min}$ and maintained constant until $9 \mathrm{~min}$. Danofloxacin, difloxacin, enrofloxacin, flumequine, and sarafloxacin levels were analyzed through gradient elution by using the A2 eluent $(0.1 \% \mathrm{FA})$ and $\mathrm{B} 2$ eluent $(100 \% \mathrm{MeOH})$. A mobile phase gradient was started at $10 \%$ B1 for $1 \mathrm{~min}$ at a flow rate of $0.5 \mathrm{~mL} / \mathrm{min}$, linearly increased to $95 \% \mathrm{~B} 1$ at $4 \mathrm{~min}$, and subsequently maintained constant until $8 \mathrm{~min}$. Thereafter, it was changed to $10 \% \mathrm{~B} 1 \mathrm{after}$ $8.1 \mathrm{~min}$ and maintained constant until $9 \mathrm{~min}$. The A3 eluent $(0.005 \mathrm{M}$ ammonium acetate in $0.1 \% \mathrm{FA})$ and $\mathrm{B} 2$ eluent $(0.005 \mathrm{M}$ ammonium acetate in $\mathrm{MeOH})$ were used as the mobile phase for nitrofuran metabolite analysis. The mobile phase gradient was started with $30 \% \mathrm{~B} 2$ at a flow rate of $0.3 \mathrm{~mL} / \mathrm{min}$, increased linearly to $95 \%$ B2 in 4 min, further maintained until $6 \mathrm{~min}$, subsequently changed to $30 \% \mathrm{~B} 2$ after $7 \mathrm{~min}$, and maintained constant until $10 \mathrm{~min}$. The A4 eluent ( $0.1 \% \mathrm{FA})$ and $\mathrm{B} 4$ eluent $(\mathrm{MeOH})$ were used as the mobile phase for MG and LMG analyses, respectively. These dyes were separated following the gradient program. The mobile phase gradient was started with $10 \% \mathrm{~B} 4$ for $1 \mathrm{~min}$ at a flow rate 
of $0.5 \mathrm{~mL} / \mathrm{min}$, increased from $10 \%$ to $95 \%$ B4 in $4 \mathrm{~min}$, and maintained constant until 8 min. Finally, B4 was changed to $10 \%$ after $8.1 \mathrm{~min}$ and maintained constant until $11 \mathrm{~min}$. The MS source conditions in ABI 4000 QTRAP were as follows: ion spray voltage of $4.5-5.5 \mathrm{kV}$, curtain gas of 15 psi, nebulizer gas of $50 \mathrm{psi}$, auxiliary gas of $60 \mathrm{psi}$, and source temperature of $50{ }^{\circ} \mathrm{C}$. MS/MS experiments were conducted in multiple reaction monitoring modes (MRMs) for simultaneous detection of all targets, with two precursor-to-product ion transitions monitored for each analyte. The mass spectrometer was set to detect negative and positive ESI interface modes for chloramphenicol and other veterinary drugs, respectively. Supplementary Table S1 lists the retention times and the precursor and corresponding product ions obtained through MRM detection in LC-amenable veterinary analytes. The dwell time for each MRM transition was set at $5 \mathrm{~ms}$. Analyst software (version 1.4, Applied Biosystems, Foster City, CA, USA) was used for instrument control and data acquisition.

\subsection{Preparing the Standard Solutions}

In volumetric flasks, stock solutions were prepared that contained pesticide standards or individual veterinary drugs by dissolving each analyte $(100 \mathrm{mg})$ in $100 \mathrm{~mL}$ of-depending on the solubility of the analytes- $\mathrm{ACN}$, acetone, or $\mathrm{MeOH}$. All types of stock solution were combined and diluted to $1 \mathrm{mg} / \mathrm{L}$ to obtain a working standard mixture. We stored all solutions at $-20^{\circ} \mathrm{C}$, and before use, a solution was allowed to adjust to room temperature. With these working standard solutions, serial dilution was performed to prepare a series of calibration standards (dilution range $0.5-500 \mathrm{ng} / \mathrm{mL}$ ).

\subsection{Extraction Procedure and Analysis}

To detect residues of chloramphenicol classes, MG, LMG, and quinolone classes, we extracted and cleaned each shrimp sample by using a modification of the veterinary drug residue analysis technique reported by Chang et al. [5] and Smith et al. [8] for aquatic products. Briefly, $2 \mathrm{~g}$ of sample was weighed in a propylene centrifuge tube (volume $50 \mathrm{~mL}$ ) and transferred to a homogenizer containing $100 \mu \mathrm{L}$ of internal standards $(100 \mathrm{ng} / \mathrm{mL}), 50 \mu \mathrm{L}$ of TMPD, and $10 \mathrm{~mL}$ of ACN. Then, we added $5 \mathrm{~mL}$ of $\mathrm{n}$-hexane saturated with CAN to the homogenate, which was shaken in a vortex mixer for $5 \mathrm{~min}$, followed by centrifugation at $4500 \mathrm{rpm}$ for $10 \mathrm{~min}$. We aspirated and subsequently discarded the hexane layer. The ACN extraction layer was collected and dried at $40{ }^{\circ} \mathrm{C}$ in a nitrogen evaporator. We re-extracted the remaining tissue pellets using $10 \mathrm{~mL}$ of $\mathrm{ACN}$ and $5 \mathrm{~mL}$ of $\mathrm{ACN}$-saturated n-hexane and then centrifuged them. The first extract was combined with the ACN layer. Subsequently, we evaporated the combined extracts to dryness at $0.5 \mathrm{~mL}$. An additional $0.5 \mathrm{~mL}$ of ultrapure water was added, after which the vortex was mixed and then sonicated for $1 \mathrm{~min}$. The reconstituted extracts underwent centrifugation at $4500 \mathrm{rpm}$ for $5 \mathrm{~min}$. Finally, a $0.2 \mu \mathrm{m}$ polyvinylidene fluoride filter (Whatman, Maidstone, UK) was employed to filter the supernatant layer, and the filtrate was transferred to an autosampler vial prior to being injected into the chromatographic system.

Nitrofuran metabolite extraction from samples was performed through the execution of a TFDA-procedure-based method [9]. Briefly, in a centrifuge tube measuring $50 \mathrm{~mL}$ in volume, we fortified $2 \mathrm{~g}$ of a sample with $100 \mu \mathrm{L}$ of internal standards $(100 \mathrm{ng} / \mathrm{mL})$, followed by sequentially adding $0.125 \mathrm{M} \mathrm{HCl}(9 \mathrm{~mL})$ and $50 \mathrm{mM}$ 2-NBA in $\mathrm{MeOH}(400 \mu \mathrm{L})$. Samples were vortex mixed (1 min), followed by overnight incubation $\left(16 \mathrm{~h}, 37^{\circ} \mathrm{C}\right)$ with gentle shaking in a water bath. In order to neutralize the samples, we added $0.8 \mathrm{M} \mathrm{NaOH}(1 \mathrm{~mL})$ and $0.1 \mathrm{M}$ dipotassium phosphate buffer $(1 \mathrm{~mL})$, and we adjusted the reaction mixture to $\mathrm{pH} 7.1-7.5$. The mixture underwent $1 \mathrm{~min}$ of vigorous vortex mixing and was then centrifuged at $3500 \mathrm{rpm}$ for $5 \mathrm{~min}$. After the collection of the supernatant, the remaining tissue pellet was re-extracted using ultrapure water $(3 \mathrm{~mL})$, as described earlier in the text, and centrifuged again. The combined extracts were re-extracted using $0.5 \mathrm{~g}$ of $\mathrm{NaCl}$ and $12 \mathrm{~mL}$ of EtOAc with vortex shaking of the samples for $1 \mathrm{~min}$. The reconstituted extracts were again centrifuged for $5 \mathrm{~min}$ at $3500 \mathrm{rpm}$. The solvent was evaporated at $40{ }^{\circ} \mathrm{C}$ in a nitrogen evaporator. We reconstituted the resultant dry extract in $1 \mathrm{~mL}$ of $50 \% \mathrm{MeOH}$, after which it was vortex mixed for $1 \mathrm{~min}$. Subsequently, $1 \mathrm{~mL}$ of $\mathrm{n}$-hexane was added to the extracts, which then underwent centrifugation again, as described 
earlier. The lower layer was collected and filtered ( $0.2 \mu \mathrm{m}$ filter membrane). The filtrate was placed in an autosampler vial prior to analysis.

\subsection{Assurance and Validation of Quality}

We validated the proposed method by calculating the recovery, linearity range, repeatability, and limits of quantification (LOQs) [10,11]. For determining the recovery and repeatability, we spiked blank samples in triplicate by using the following standard mixture of analytes at two concentrations (low and high levels): 1 and $5 \mathrm{ng} / \mathrm{g}$ for determination of chloramphenicol classes, dyes, and nitrofuran metabolites; and 5 and $25 \mathrm{ng} / \mathrm{g}$ for determination of quinolone classes. Extraction and treatment of the samples followed a previously reported protocol $[2,8,9]$. The aforementioned recovery validation method was employed to determine the method's repeatability, which was calculated as the percentage of the relative standard deviation (RSD\%). The recovery and repeatability (expressed as the percentage of relative standard deviation) of veterinary drugs ranged from $88.67 \%$ to $92.35 \%$ (repeatability range: $3.79-9.67 \%$ ) for chloramphenicol classes, $75.21 \%$ to $103.31 \%$ (repeatability range: $6.72-14.58 \%$ ) for quinolone classes, $98.81 \%$ to $100.31 \%$ (repeatability range: $3.58-8.13 \%$ ) for MG and LMG, and $99.29 \%$ to $100.52 \%$ (repeatability range: $0.98-5.58 \%$ ) for nitrofuran metabolites in shrimp samples (Supplementary Table S2). Matrix-matched calibration executed through the use of blank sample extracts and addition of the corresponding amount of working solution (with target compounds at a concentration of $0.5-500 \mathrm{ng} / \mathrm{mL}$ ) was performed to evaluate the linearity. The calibration curves obtained had high linearity and reproducibility, with the analytical matrix-matched calibration achieving favorable correlation coefficients $\left(R^{2}>0.990\right)$. The LOQs were defined as being the concentrations of analyte that yielded peak signals $3 \times$ and $10 \times$ the intensity of background noise from the chromatogram. The florfenicol, thiamphenicol, chloramphenicol, LMG, MG, and nitrofuran metabolite LOQ was $0.25 \mathrm{ng} / \mathrm{mL}$ in shrimp samples. Compared with these chemicals, the LOQ of other veterinary drugs, including danofloxacin, difloxacin, enrofloxacin, flumequine, and sarafloxacin, was $1 \mathrm{ng} / \mathrm{g}$ (Supplementary Table S2); concentrations lower than these LOQs indicated that the chemicals and drugs were considered undetectable.

\subsection{EDI}

To assess the degree to which people are exposed to veterinary drug residues in shrimp, we estimated the EDI from the residual veterinary drug concentrations. The acceptable daily intakes (ADIs) established by the World Health Organization (WHO) and Food and Agriculture Organization of the United Nations (FAO) were employed as points of comparison. The following equation was used to calculate the EDI: EDI $(\mathrm{ng} / \mathrm{kg} /$ day $)=($ daily fish consumption $[\mathrm{g} /$ day $]) \times($ mean veterinary drug concentration [ng/g])/(human body weight [kg]) [6]. Data regarding Taiwanese citizens' daily seafood consumption ( $96.9 \mathrm{~g}$ for men and $74.2 \mathrm{~g}$ for women) were collected from the National Nutrition and Health Survey conducted by the Ministry of Health and Welfare [12]. We considered the mean Taiwanese body weight to be $60 \mathrm{~kg}$ [12]. We determined the maximal EDI from the maximum residue concentrations.

\section{Results}

\subsection{Detection Rates and Levels of Veterinary Drugs in Shrimp Samples}

In total, 23 whiteleg, 16 grass, and 14 giant river shrimp samples were collected. Chloramphenicol was detected in one grass shrimp, enrofloxacin in one whiteleg shrimp, and flumequine in one whiteleg shrimp and one giant river shrimp (Table 1). These detected veterinary drugs are prohibited by the TFDA for use in shrimp. In all shrimp samples, the predominant residue was flumequine at $3.77 \%$ (2/53), followed by chloramphenicol at $1.89 \%(1 / 53)$ and enrofloxacin at $1.89 \%(1 / 53)$. Veterinary drugs were detected in $8.70 \%(2 / 23), 6.25 \%(1 / 16)$, and $7.14 \%(1 / 14)$ of the whiteleg, grass, and giant river shrimp samples, respectively. The levels of chloramphenicol and enrofloxacin were 0.29 and $5.62 \mathrm{ng} / \mathrm{g}$ 
in one grass and whiteleg shrimp, respectively. Moreover, flumequine (1.01-1.52 ng/g) was detected in two shrimp samples, namely in one whiteleg and one giant river shrimp. The concentrations of chloramphenicol, enrofloxacin, and flumequine (derived from all samples, including samples with detected and undetected concentrations) were $0.01,0.11$, and $0.05 \mathrm{ng} / \mathrm{g}$, respectively. Overall, $7.55 \%$ (4/53) of all shrimp samples contained detectable veterinary drug residues, which indicated the positive ratio of banned residual drugs.

Table 1. Detection levels of banned veterinary drugs in various shrimp samples collected between July 2016 and December 2017.

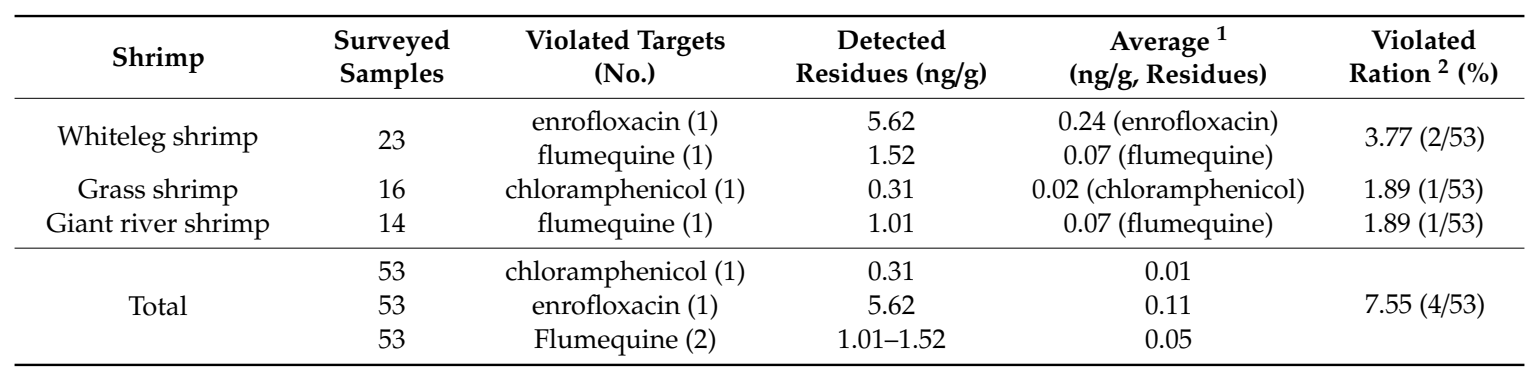

${ }^{1}$ Estimated from all samples, including samples with detected and undetected concentrations. ${ }^{2}$ Samples with residual concentrations lower than the LOQ were considered to have undetectable concentrations.

\subsection{EDIs of Taiwanese Adults for Veterinary Drug Residues in Shrimp Samples}

The Joint FAO/WHO Expert Committee on Food Additives (JECFA) determined the inappropriateness of establishing a chloramphenicol ADI [13]. Therefore, we did not estimate the EDI of chloramphenicol residues in shrimp samples. The EDIs calculated from the average enrofloxacin and flumequine levels were, respectively, 0.14 and $0.06 \mathrm{ng} / \mathrm{kg}$ body weight/day for women and 0.18 and $0.08 \mathrm{ng} / \mathrm{kg}$ body weight/day for men (Table 2). Regarding the veterinary drug residues in food, the ADIs stipulated by the JECFA's Joint Meeting of the FAO/WHO for enrofloxacin and flumequine are 0.002 and $0.03 \mathrm{mg} / \mathrm{kg}$, respectively [14,15]. As detailed in Table 2, the obtained EDIs were considerably lower than the enrofloxacin and flumequine ADIs recommended by the FAO/WHO. For enrofloxacin and flumequine, the EDIs expressed as a percentage of the ADIs were, respectively, $0.01 \%$ and $0.0003 \%$ for men and $0.01 \%$ and $0.0002 \%$ for women. Overall, consumption of shrimp lead to a low risk of dieldrin exposure, with the ADIs lower than $1.0 \%$ for men and women.

Table 2. Estimated dietary intake of quinolone residues in Taiwanese adults.

\begin{tabular}{|c|c|c|c|c|c|}
\hline \multirow{2}{*}{ OCPs } & \multicolumn{2}{|c|}{$\begin{array}{c}\text { EDI } \\
\text { (ng/kg Body Weight/Day) }\end{array}$} & \multicolumn{2}{|c|}{ EDI\% of ADI } & \multirow{2}{*}{$\begin{array}{c}\text { ADI (FAO/WHO) } \\
\text { (mg/kg Body Weight/Day) }\end{array}$} \\
\hline & Male & Female & Male & Female & \\
\hline Enrofloxacin & 0.18 & 0.14 & 0.01 & 0.01 & 0.002 \\
\hline Flumequine & 0.08 & 0.06 & 0.0003 & 0.002 & 0.03 \\
\hline
\end{tabular}

\section{Discussion}

In the present study, 14 residual veterinary drugs, namely three chloramphenicol classes (chloramphenicol, florfenicol, and thiamphenicol), five quinolone classes (danofloxacin, difloxacin, enrofloxacin, flumequine, and sarafloxacin), MG, LMG, and four nitrofuran metabolites (AMOZ, AOZ, $\mathrm{AH}$, and SC), were analyzed in 52 shrimp samples collected from aquaculture areas in Taiwan. To validate the presence of these compounds in samples, we evaluated the mean recovery (as a measure of trueness), linearity, sensitivity, and repeatability (as a measure of precision) according to EU guidelines (SANCO/12495/2011) [10]. Because chloramphenicol, nitrofuran metabolites, MG, and LMG are banned from use in edible animals and danofloxacin, difloxacin, enrofloxacin, flumequine, and sarafloxacin are banned from use in decapods, the TFDA does not recommend MRLs in shrimp. 
Therefore, the residues of these banned compounds in shrimp were detected and sufficiently indicated the degree of legal compliance regarding the use of these products.

The Commission Decision 2002/657/EC criteria for evaluation of veterinary drug residues in animals and animal products are stipulated on the basis of mass spectrometry at numerous identification points (IPs) [16]. Source conditions were optimized to obtain 1.5 IP from product ions and one IP from precursor ions for each compound. In general, obtaining four IPs at the lowest level is required for analyzing banned compounds. In the present study, veterinary drugs were analyzed in the MRM mode by monitoring three different ions (one precursor and two fragment ions). Using this approach, we achieved four IPs (one IP from a single precursor ion and three IPs from two fragment ions), as mandated by the aforementioned guidelines. Our analysis method successfully identified the residues of MG, LMG, chloramphenicol classes, quinolone classes, and nitrofuran metabolites.

The analytical extraction method for aquatic samples was designed by Smith et al. [8]. In this method, ACN and hexane are used to extract samples for simultaneously screening multiple classes of drug residues, including macrolides, $\beta$-lactam antibiotics, dyes, quinolones, tetracyclines, and antimycotic imidazoles. Moreover, other extraction methods have been reported for analyzing chloramphenicol [17], MG, and LMG [18]. In addition, we applied this method for the extraction of chloramphenicol, MG, and LMG residues in bivalve samples [5]. The method used herein was developed for the simultaneous detection of chloramphenicol classes, quinone classes, MG, and LMG in shrimp samples. This is the most efficient and energy-conservative method for veterinary drug extraction. However, the same method could not be employed to analyze the nitrofuran metabolite residues in aquatic samples; because of their chemical structural characteristics, nitrofuran metabolites in food samples were extracted using 2-NBA for derivatization [19].

To validate the shrimp sample analysis method, as recommended by the TFDA [11], the acceptable recovery rate had to be $70-120 \%$ with RSD $<15 \%$ for chemical residues in food matrixes detected in the $0.1-1 \mathrm{mg} / \mathrm{kg}$ range; $70-120 \%$ with RSD $<20 \%$ for those detected in the $0.01-0.1 \mathrm{mg} / \mathrm{kg}$ range; $60 \%-125 \%$ with RSD $<30 \%$ for those detected in the $0.001-0.01 \mathrm{mg} / \mathrm{kg}$ range; and $50-125 \%$ with RSD $<35 \%$ for those detected within $\leq 0.001 \mathrm{mg} / \mathrm{kg}$. According to our results, veterinary drug residues detected within $\leq 0.001 \mathrm{mg} / \mathrm{kg}$ and $0.001-0.01 \mathrm{mg} / \mathrm{kg}$ ranges demonstrated a recovery rate of $80-120 \%$ with an RSD of $<10 \%$ and a recovery rate of $70-120 \%$ with an RSD of $<15 \%$, respectively. The TFDA also recommends various LOQs, including $0.3 \mathrm{ng} / \mathrm{g}$ in chloramphenicol; $5 \mathrm{ng} / \mathrm{g}$ in florfenicol and thiamphenicol; $10 \mathrm{ng} / \mathrm{g}$ in quinolone classes; $0.5 \mathrm{ng} / \mathrm{g}$ in MG and LMG; and $1 \mathrm{ng} / \mathrm{g}$ in nitrofuran metabolites, for aquatic food for the assessment of veterinary drug residues in seafoods [20]. Compared with the LOQs recommended by the TFDA, the LOQs obtained using our analytical method were lower and can be employed to detect trace veterinary drug residues. Therefore, the analytical methods employed herein conform to the recommendations of the TFDA.

The regulations entitled Tolerances for Residues of Veterinary Drugs in Food, established by the Ministry of Health and Welfare of Taiwan, state that nitrofuran metabolites, chloramphenicol, MG, and LMG are banned from use in shrimp culturing because of concerns that pertain to mutagenicity and carcinogenicity [5]. In addition, food-producing animals and products containing these chemicals exported by third-world countries are prohibited in Japan and the European Union, the major importers of Taiwanese marine products. Based on methodologies available for detecting banned compounds in edible products, the Department of Health of Taiwan [21] and EU Commission [22] have both established a maximum residual permissible limit (MRPL) of $1 \mathrm{ng} / \mathrm{g}$ for each nitrofuran metabolite in aquaculture, marine, and poultry meat products. Furthermore, the EU Commission stipulates an MRPL of $0.3 \mathrm{ng} / \mathrm{g}$ for chloramphenicol and $2 \mathrm{ng} / \mathrm{g}$ for MG plus LMG [16] in all food products of animal origin to ensure that customers worldwide are given the same level of protection. According to the aforementioned guidelines, the LOQs of our methods executed for identifying the levels of chloramphenicol, dye, and nitrofuran metabolite residues in shrimp meet the MRPL.

The chromatography-mass spectrometry screening of carcinogenic antimicrobials-such as nitrofuran metabolites, chloramphenicol, MG, and LMG—in 53 shrimp samples demonstrated a positive 
result, with the chloramphenicol concentration being greater than the MRPL of chloramphenicol set by the EU Commission ( $0.31 \mathrm{ng} / \mathrm{g}$ in a grass shrimp sample). Administering chloramphenicol to food-producing animals is banned in Taiwan. Some aquaculture farmers use chloramphenicol regardless, however, because it is a broad-spectrum, inexpensive, and readily available antibiotic [23]. Mixed breeding has caused chloramphenicol to be employed for the prevention as well as treatment of infectious diseases in shrimp. In the present study, the proportion of positive identification of banned veterinary drugs was $1.89 \%$ (1/53). In the analysis of cultured shrimp in Bangladesh, the detection of chloramphenicol and nitrofuran metabolite residues revealed a violation ratio of $8.37 \%(118 / 1409)$ in $2008,8.16 \%$ (182/2230) in 2009, and 5.81\% (122/2098) in 2009 [24]. In addition, in the Canadian Total Diet Study from 1994 to 2004, the detection of MG, LMG, and nitrofuran metabolite residues revealed a violation ratio of $20.0 \%$ (6/30) [25]. In Ireland, exposure to nitrofuran residues was assessed from 2009 to 2010 , which revealed a violation ratio of $5.68 \%$ (5/88) in the detection of SEM residues [26]. However, in the aforementioned reports, only two classes of nitrofuran metabolites and chloramphenicol or three classes of MG, LMG, and nitrofuran metabolites were detected. Our present findings differ from those of TFDA surveys. In reports in recent years, the violation ratio of banned veterinary drug residues in shrimp samples was $0 \%$ in $2013(0 / 20)$ [27] and $0 \%$ in 2014 (0/20) [28]. These differences are partially accounted for by varying sample sizes. In addition, the samples collected in this study were obtained from shrimp production areas in Taiwan, whereas the samples collected by the TFDA may have been imported shrimp. Therefore, several categories of banned veterinary drugs in Taiwanese shrimp were appropriately detected in the present study.

In the present investigation, quinolone residues (3/53) were detected with a higher violation ratio than chloramphenicol $(1 / 53)$ in aquaculture shrimp. Our study revealed that quinolones were the predominant compounds in the aquacultured shrimp samples in Taiwan, which was similar to the results of a survey conducted in China [29], Vietnam [30,31], and Thailand [31] following intensive use in aquaculture to treat bacterial infections, which polluted aquatic habitats and had adverse effects on the health of freshwater organisms. Quinolones were detected in $8.70 \%(2 / 23)$ and $7.14 \%(1 / 14)$ of whiteleg and giant river shrimp samples, respectively. In all shrimp samples, the predominant residue was flumequine at $3.77 \%$ (2/53), followed by enrofloxacin at $1.89 \%(1 / 53)$. The results of the present study are similar to those of the survey conducted by the TFDA. Compared with a report of the TFDA in 2012, the violation ratio of quinolone residues in shrimp samples was $4.0 \%$ in 25 samples, which was positive with flumequine at $21.0 \mathrm{ng} / \mathrm{g}$ in one shrimp sample [32]. From the data available, we concluded that flumequine continues to be used as a growth promoter and prophylactic agent in aquatic products because of its affordability and effectiveness. Other surveys in Asia $[31,33]$ have reported that flumequine has been the most widely used synthetic antibiotic in aquaculture, especially because of its relative stability to resist bacterial degradation in water and sediments. In addition, flumequine residues were detected in trace amounts; only a concentration of 1.01-1.52 ng/g or higher triggers action by the TFDA (withdrawal of the product and issuance of an alert). The results of the surveys reviewed herein indicate that the Taiwanese population is exposed to trace amounts of flumequine that do not pose an immediate risk to health through the consumption of shrimp. Therefore, Taiwan's regulatory authorities and producers should continually monitor aquatic products and prevent sources of contamination, ensuring the chemical safety of commercially available foods.

Parameter guidelines indicate how the risk to organisms such as humans can be assessed by stipulating criteria related to the ADI, hazard quotients, provisional tolerable weekly intake, and excess cancer risk $[6,34,35]$. Guidelines for the ADI, such as those formulated by the FAO and WHO, facilitate the assessment of risks to organisms, including humans [6]. The ADI is a single value, however, and eating habit and consumption rate differences are not considered in its calculation [36,37]. The JECFA [38] and US EPA [39] have proposed a new and highly accurate measure for the estimation of chronic dietary intake: the EDI. In this study, we concluded that the ADI was not exceeded by the corresponding daily exposure. Because few residual quinolones were discovered, the estimated EDI revealed that consumption of the investigated shrimp would result in considerably less dietary 
intake of enrofloxacin and flumequine in the Taiwanese population than that stipulated by the ADI. Furthermore, when assessed against the ADIs, the EDIs calculated in this study indicated no risk to health due to shrimp consumption. The EDIs were lower than $1 \%$ of the ADIs in this study, indicating negligible risk $[6,38]$. Thus, the levels of quinolone in Taiwanese food products can be concluded to not negatively affect health. Because of the potentially adverse effects of antibiotics on health and aquatic environments, the impact of these pollutants must be urgently evaluated further.

\section{Conclusions}

In the present study, we analyzed the residues of chloramphenicol, florfenicol, thiamphenicol, MG, LMG, nitrofuran metabolites, danofloxacin, difloxacin, enrofloxacin, flumequine, and sarafloxacinthe in shrimp samples; methods used were validated according to the EU criteria and complied with the MRPLs established by the EU and TFDA. The residues of banned veterinary drugs chloramphenicol and quinolone, with no MRL recommended, were detected in 53 shrimp samples. We observed that one shrimp sample contained chloramphenicol, one shrimp sample contained enrofloxacin, and two shrimp samples contained flumequine. Notably, only trace amounts of all residues were discovered, indicating no immediate risk to health because the EDIs were considerably lower than the FAO/WHO-defined ADIs. Enrofloxacin and flumequine contamination following shrimp consumption in Taiwan appears to present a negligible threat to human health. However, the concern regarding pharmaceuticals and their adverse effects on the environment and human health is increasing, and a background information system on the consumption of veterinary antibiotics through shrimp must be established and improved, thus providing a monitoring and management framework. The health and agricultural authorities can use the present study findings as a valuable reference when improving contaminant regulation in aquaculture.

Supplementary Materials: The following are available online at http://www.mdpi.com/2076-3417/9/12/2463/s1, Supplementary Table S1: Retention time and MS/MS fragmentation conditions for veterinary drugs and their corresponding internal standards, Supplementary Table S2: Recovery, repeatability, and LOQ of veterinary drugs spiked into whiteleg shrimp.

Author Contributions: M.-Y.T. and C.-F.L. conceived the idea and performed experiments. W.-C.Y., C.-T.L., and K.-H.H. assisted in recombinant construction. G.-R.C. wrote, reviewed, and edited the manuscript. M.-Y.T. and C.-F.L. contributed equally to this work.

Funding: This research received no external funding.

Acknowledgments: This study was supported by the Ministry of Science and Technology (Taiwan) (MOST 107-2313-B-415-012) and, in part, by the Taichung Veterans General Hospital (Taiwan) and National Chung-Hsing University (Taiwan) (TCVGH-NCHU-10776013). This manuscript was edited by Wallace Academic Editing.

Conflicts of Interest: The authors declare no conflict of interest.

\section{References}

1. Chang, G.R. Surveys on banned veterinary drugs residues in marine bivalves and gastropods in Taiwan between 2010 and 2015: A mini review. J. Aquat. Pollut. Toxicol. 2017, 1, 1-5.

2. Liao, I.C. Aquaculture practices in Taiwan and its visions. J. Fish. Soc. Taiwan 2005, 32, 193-206.

3. Fisheries Agency, Council of Agriculture, Taiwan. 2017 Fisheries Statistical Yearbook. 2018. Available online: https://www.fa.gov.tw/cht/PublicationsFishYear/ (accessed on 5 February 2019).

4. Chen, C.L.; Qiu, G.H. The long and bumpy journey Taiwan's aquaculture development and management. Mar. Policy 2014, 48, 152-161. [CrossRef]

5. Chang, G.R.; Chen, H.S.; Lin, F.Y. Analysis of banned veterinary drugs and herbicide residues in shellfish by liquid chromatography-tandem mass spectrometry (LC/MS/MS) and gas chromatography-tandem mass spectrometry (GC/MS/MS). Mar. Pollut. Bull. 2016, 113, 579-584. [CrossRef] [PubMed]

6. Chang, G.R. Persistent organochlorine pesticides in aquatic environments and fishes in Taiwan and their risk assessment. Environ. Sci. Pollut. Res. Int. 2018, 25, 7699-7708. [CrossRef] [PubMed] 
7. Song, K.H.; Breslin, V.T. Accumulation and transport of sediment metals by the vertically migrating opossum shrimp, Mysis relicta. J. Gt. Lakes Res. 1999, 25, 429-442. [CrossRef]

8. Smith, S.; Gieseker, C.; Reimschuessel, R.; Decker, C.S.; Carson, M.C. Simultaneous screening and confirmation of multiple classes of drug residues in fish by liquid chromatography-ion trap mass spectrometry. J. Chromatogr. A 2009, 1216, 8224-8232. [CrossRef] [PubMed]

9. Department of Health, Executive Yuan, Taiwan. Method of Test for Veterinary Drug Residues in Foods-Test of Nitrofuran Metabolites; Announcement No. 1021950758; Department of Health, Executive Yuan, Taiwan: Taipei, Taiwan, 2013.

10. European Union. Method Validation and Quality Control Procedures for Pesticides Residues Analysis in Food and Feed; Document No SANCO/12495/2011; European Union: Brussels, Belgium, 2011.

11. Shen, Y.R.; Cheng, M.W.; Wu, B.S.; Yang, K.C.; Chang, Y.H.; Tseng, S.H.; Kao, Y.M.; Chiueh, L.C.; Shih, D.Y.C. Developement of the method for analysis of multiple pesticide residues in animal matrices by QuEChERS method. Taiwanese. J. Agric. Chem. Food Sci. 2013, 51, 148-160.

12. Wu, S.J.; Chang, Y.H.; Fang, C.W.; Pan, W.H. Food sources of weight, calories, and three macro-nutrientsNAHSIT 1993-1996. Nutr. Sci. J. 1999, 24, 41-58.

13. Nicolich, R.S.; Werneck-Barroso, E.; Marques, M.A.S. Food safety evaluation: Detection and confirmation of chloramphenicol in milk by high performance liquid chromatography-tandem mass spectrometry. Anal. Chim. Acta 2006, 565, 97-102. [CrossRef]

14. He, X.T.; Deng, M.C.; Wang, Q.; Yang, Y.T.; Yang, Y.F.; Nie, X.P. Residues and health risk assessment of quinolones and sulfonamides in cultured fish from Pearl River Delta, China. Aquaculture 2016, 458, 38-46. [CrossRef]

15. Itoh, T.; Mitsumori, K.; Kawaguchi, S.; Sasaki, Y.F. Genotoxic potential of quinolone antimicrobials in the in vitro comet assay and micronucleus test. Mutat. Res. 2006, 603, 135-144. [CrossRef] [PubMed]

16. European Commission. Directive 2002/657/EC concerning the performance of analytical methods and the interpretation of results. Off. J. Eur. Commun. 2002, L221, 8-36.

17. Takinoa, M.; Daishimab, S.; Nakaharac, T. Determination of chloramphenicol residues in fish meats by liquid chromatography-atmospheric pressure photoionization mass spectrometry. J. Chromatogr. A 2003, 1011, 67-75. [CrossRef]

18. Lee, K.C.; Wu, J.L.; Cai, Z. Determination of malachite green and leucomalachite green in edible goldfish muscle by liquid chromatography-ion trap mass spectrometry. J. Chromatogr. B Anal. Technol. Biomed. Life Sci. 2006, 843, 247-251. [CrossRef] [PubMed]

19. Radovnikovic, A.; Moloney, M.; Byrne, P.; Danaher, M. Detection of banned nitrofuran metabolites in animal plasma samples using UHPLC-MS/MS. J. Chromatogr. B Anal. Technol. Biomed. Life Sci. 2011, 879, 159-166. [CrossRef] [PubMed]

20. Fu, H.P.; Chen, C.M.; Hang, S.F.; Lin, Y.Y.; Lin, Y.R.; Chen, T.L. Post-market surveillance study on veterinary drug residues in poultry, livestock and aquatic products in 2017. Ann. Rep. Food Drug Res. 2018, 9, 115-124.

21. Ministry of Health and Welfare, Executive Yuan, Taiwan. Tolerances for Residues of Veterinary Drugs; MOHW Food No. 1041303515; Ministry of Health and Welfare, Executive Yuan, Taiwan: Taipei, Taiwan, 2015.

22. European Commission. Decision 2003/181/EC amending decision 2002/657/EC as regards the setting of minimum performance limits (MRPLs) for certain residues in food animal origin. Off. J. Eur. Commun. 2013, L71, 17.

23. Defoirdt, T.; Sorgeloos, P.; Bossier, P. Alternatives to antibiotics for the control of bacterial disease in aquaculture. Curr. Opin. Microbiol. 2011, 14, 251-258. [CrossRef] [PubMed]

24. Hassan, M.N.; Rahman, M.; Hossain, M.B.; Hossain, M.M.; Mendes, R.; Nowsad, A.A.K.M. Monitoring the presence of chloramphenicol and nitrofuran metabolites in cultured prawn, shrimp and feed in the Southwest coastal region of Bangladesh. Egypt. J. Aquat. Res. 2013, 39, 51-58. [CrossRef]

25. Tittlemier, S.A.; Riet, J.; Burns, G.; Potter, R.; Murphy, C.; Rourke, W.; Pearce, H.; Dufresne, G. Analysis of veterinary drug residues in fish and shrimp composites collected during the Canadian Total Diet Study, 1993-2004. Food Addit. Contam. 2007, 24, 14-20. [CrossRef]

26. Radovnikovic, A.; Conroy, E.R.; Gibney, M.; O’Mahony, J.; Danaher, M. Residue analyses and exposure assessment of the Irish population to nitrofuran metabolites from different food commodities in 2009-2010. Food Addit. Contam. A 2013, 30, 1858-1869. [CrossRef] [PubMed] 
27. Fu, H.P.; Kuo, H.W.; Shih, C.C.; Lin, H.C.; Lin, K.H.; Lin, Y.R.; Chou, H.K.; Shyu, J.F.; Pan, J.Q.; Hsu, C.K.; et al. 2013 Post-market survey on veterinary drug residues in livestock and aquatic products. Ann. Rep. Food Drug Res. 2014, 5, 81-91.

28. Fu, H.P.; Lin, Y.P.; Su, H.C.; Wang, T.S.; Hsu, C.H.; Liu, F.M.; Feng, R.L.; Hsu, C.K.; Liu, L.W.; Tzeng, G.S.; et al. 2014 Post-market survey on veterinary drug residues in livestock and aquatic products. Ann. Rep. Food Drug Res. 2015, 6, 67-75.

29. Zhao, S.; Jiang, H.; Li, X.; Mi, T.; Li, C.; Shen, J. Simultaneous determination of trace levels of 10 quinolones in swine, chicken, and shrimp muscle tissues using HPLC with programmable fluorescence detection. J. Agric. Food Chem. 2007, 55, 3829-3834. [CrossRef] [PubMed]

30. Pham, D.K.; Chu, J.; Do, N.T.; Brose, F.; Degand, G.; Delahaut, P.; De Pauw, E.; Douny, C.; Nguyen, K.V.; $\mathrm{Vu}, \mathrm{T} . \mathrm{D}$; et al. Monitoring antibiotic use and residue in freshwater aquaculture for domestic use in vietnam. Ecohealth 2015, 12, 480-489. [CrossRef] [PubMed]

31. Takasu, H.; Suzuki, S.; Reungsang, A.; Pham, H.V. Fluoroquinolone (FQ) contamination does not correlate with occurrence of FQ-resistant bacteria in aquatic environments of Vietnam and Thailand. Microbes Environ. 2011, 26, 135-143. [CrossRef]

32. Fu, H.P.; Kuo, H.W.; Shih, C.C.; Lin, H.C.; Lin, Y.R.; Chou, H.K.; Shyu, J.F.; Pan, J.Q.; Hsu, C.K.; Liu, L.W.; et al. 2012 Post-market survey on veterinary drug residues in livestock and aquatic products. Ann. Rep. Food Drug Res. 2013, 4, 38-46.

33. Thuy, H.T.; Nga le, P.; Loan, T.T. Antibiotic contaminants in coastal wetlands from Vietnamese shrimp farming. Environ. Sci. Pollut. Res. Int. 2011, 18, 835-841. [CrossRef]

34. Gu, Y.G.; Lin, Q.; Huang, H.H.; Wang, L.G.; Ning, J.J.; Du, F.Y. Heavy metals in fish tissues/stomach contents in four marine wild commercially valuable fish species from the western continental shelf of South China Sea. Mar. Pollut. Bull. 2017, 114, 1125-1129. [CrossRef]

35. Ke, C.L.; Gu, Y.G.; Liu, Q.; Li, L.D.; Huang, H.H.; Cai, N.; Sun, Z.W. Polycyclic aromatic hydrocarbons (PAHs) in wild marine organisms from South China Sea: Occurrence, sources, and human health implications. Mar. Pollut. Bull. 2017, 117, 507-511. [CrossRef] [PubMed]

36. Yang, N.; Matsuda, M.; Kawano, M.; Wakimoto, T. PCBs and organochlorine pesticides (OCPs) in edible fish and shellfish from China. Chemosphere 2006, 63, 1342-1352. [CrossRef] [PubMed]

37. Li, X.; Nie, X.P.; Pan, D.B.; Li, G.Y. Analysis of PAEs in muscle tissue of freshwater fish from fishponds in Pearl River Delta. J. Environ. Health 2008, 25, 202-204.

38. Vragović, N.; Bazulić, D.; Njari, B. Risk assessment of streptomycin and tetracycline residues in meat and milk on Croatian market. Food Chem. Toxicol. 2011, 49, 352-355. [CrossRef] [PubMed]

39. Zhang, G.; Pan, Z.; Bai, A.; Li, J.; Li, X. Distribution and bioaccumulation of organochlorine pesticides (OCPs) in food web of Nansi Lake, China. Environ. Monit. Assess. 2014, 186, 2039-2051. [CrossRef] [PubMed] 